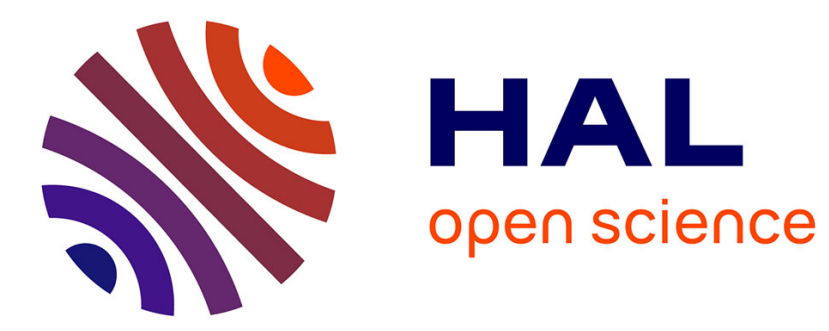

\title{
Mise en ligne d'un microscope digitalisé et télécommandé
}

\author{
P. Volmer, E. Christophel, J. Persigny, C. Jacquot
}

\section{To cite this version:}

P. Volmer, E. Christophel, J. Persigny, C. Jacquot. Mise en ligne d'un microscope digitalisé et télécommandé. Revue de Physique Appliquée, 1969, 4 (2), pp.328-328. 10.1051/rphysap:0196900402032800 . jpa-00243287

\section{HAL Id: jpa-00243287 https://hal.science/jpa-00243287}

Submitted on 1 Jan 1969

HAL is a multi-disciplinary open access archive for the deposit and dissemination of scientific research documents, whether they are published or not. The documents may come from teaching and research institutions in France or abroad, or from public or private research centers.
L'archive ouverte pluridisciplinaire HAL, est destinée au dépôt et à la diffusion de documents scientifiques de niveau recherche, publiés ou non, émanant des établissements d'enseignement et de recherche français ou étrangers, des laboratoires publics ou privés. 


\title{
MISE EN LIGNE D’UN MICROSGOPE DIGITALISÉ ET TÉLÉCOMMANDÉ
}

\author{
P. VOLMER, E. GHRISTOPHEL, J. PERSIGNY et G. JAGQUOT, \\ Laboratoire de Physique Corpusculaire, C.R.N., Strasbourg.
}

\begin{abstract}
Résumé. - On décrit la mise en ligne avec un calculateur d'un microscope digitalisé en $x, y, z$, et télécommandé, pour les mesures dans l'émulsion ionographique.

Abstract. - We describe a digitized microscope for measurements in the emulsion, on-line with a small process-control computer.
\end{abstract}

Dans le double but d'améliorer la précision et la rapidité des mesures effectuées dans l'émulsion nucléaire à l'aide d'un microscope, il a d'abord été procédé à la digitalisation des trois coordonnées. A un oculaire digitalisé décrit antérieurement [1], il a été ajouté sur la coordonnée $z$ un codeur incrémental de même type que ceux des coordonnées $x$ et $y$. La précision des mesures est ainsi supérieure à la dimension d'un grain de l'émulsion, soit $0,3 \mu \mathrm{m}$. Des compteurs totalisent les trois coordonnées $x, y, z$ d'un point visé. Les déplacements dans le champ de l'oculaire du réticule de repérage à fils croisés sont télécommandés à l'aide d'un manche à balai manipulé par l'opérateur. Pour améliorer la rapidité des mesures dans le même rapport que leur précision, et pour éviter des erreurs dans l'ordre de déroulement des mesures, l'opérateur a la possibilité d'envoyer vers un calculateur de conduite de processus (Control Data 1700) cinq types d'informations : i) un commentaire de 8 caractères numériques $\mathrm{DCB}$, indiquant par exemple le numéro de la plaque analysée, son nom, la date, etc.; ii) les trois coordonnées $x, y, z$, sous la forme de 3 chaînes de 4 caractères numériques $\mathrm{DCB}$; iii) un, parmi trois caractères alphabétiques, indiquant par exemple le type d'opération qui a été ou qui va être effectuée.

Pour permettre la mise en ligne ultérieure d'autres appareillages similaires, actuellement en cours de réalisation, la transmission des messages vers le calculateur se fait en mode interrompu caractère par caractère.

Après avoir saisi les données et commentaires, le calculateur traite ceux-ci et indique à l'opérateur, par l'intermédiaire d'un système de visualisation, la marche à suivre ultérieurement, ainsi que les erreurs éventuellement commises. Ce n'est qu'après vérification par programme de leur validité et de leur bon ordre de succession que les résultats des mesures sont, après leur mémorisation, perforés sur du ruban, en vue de leur traitement ultérieur sur gros ordinateur de calcul scientifique. La figure 1 montre l'ensemble de l'installation.

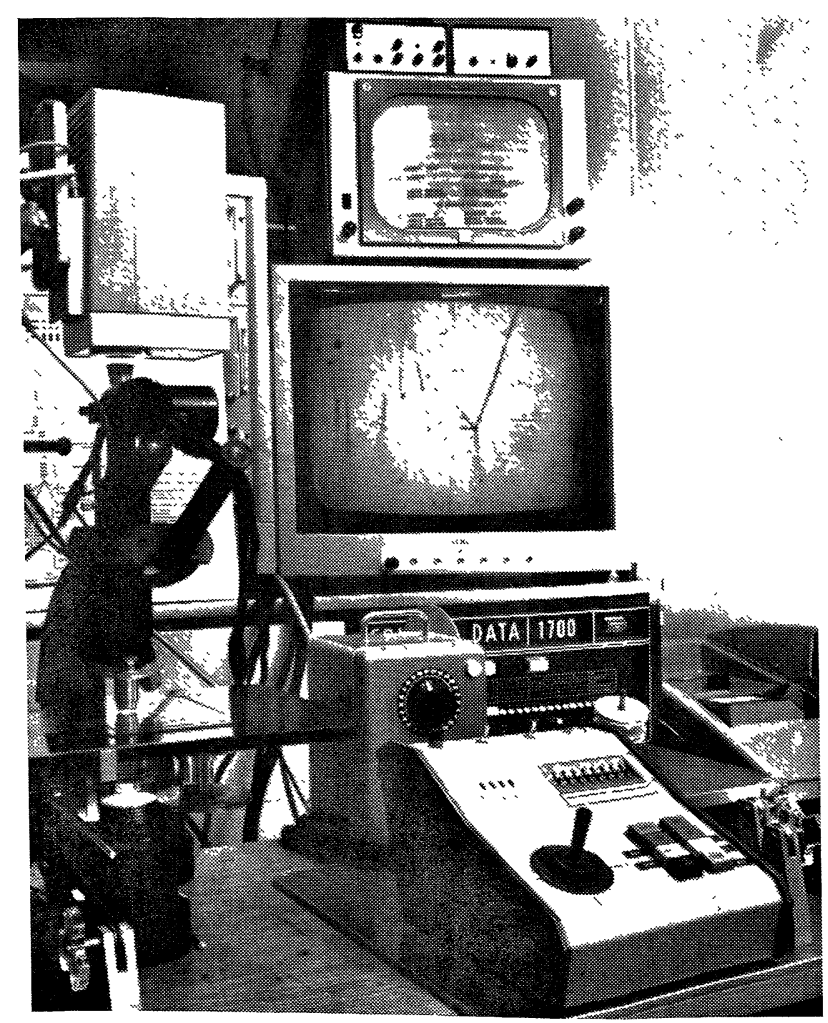

FIG. 1. - Vue de l'ensemble de l'installation.

\section{BIBLIOGRAPHIE}

[1] Volmer (P.) et Christophel (E.), Rev. Phys. Appl., 1968, 3, 116. 